\title{
Problems and Countermeasures in the Keywords of Academic Papers*
}

\author{
WANG Fengnian \\ Tsinghua University, Beijing, China
}

\begin{abstract}
Keywords with the retrieval value clearly show the main contents, enhance the influence of academic periodicals, highlight specific information, and generalize information, but because of the ignorance of the value of keywords from some authors and editors, the lack of canonical guidance, and limitations of the editorial knowledge structure, there are some problems concerning keywords in academic papers, such as papers containing too many or too few keywords, or keywords being used in a way that is too general or colloquial. According to academic principle, accurate principle, logical principle, comprehensive principle, it is imperative to discuss how to choose keywords properly.
\end{abstract}

Keywords: keywords, academic papers, selection criteria of keywords, countermeasures of keywords

Keywords, the selected words that reflect the main content of academic papers or reports, are convenient for readers who are looking for specific information. The selection of keywords therefore determines the rate at which an academic paper is found by readers when they are searching journals for information, but some authors and editors only pay attention to the academic quality of papers, neglecting the quality of their choice of keywords.

\section{The Evolution of Keywords in China}

In 1976, Bibliographic Search of Science and Technology, compiled by the Department of Science, Wuhan University Library, was published by Jilin Science and Technology Bureau (see Figure 1). In China, it was the first book to introduce the keyword index method in a separate section, which defined keywords as "meaningful vocabularies that are selected from the title, text, or abstract of a paper to characterize the matter of the document" (Wuhan University Library, 1975, pp. 53-54).

Started from 1980s, keywords were not only the study object by redactology, but also a research method in various disciplines in China. In 1987, National Bureau of Standard issued the Format of Scientific and Technical Reports, Dissertations and Academic Papers, which was named as GBT 7713-1987. As the first provision in China, GBT 7713-1987 made clear that

keywords, as an important component of scientific and technical reports, dissertations and academic papers, are selected from the report or paper for the purpose of the indexing work to indicate the contents of the full-text. Each report or paper should select 3-8 keywords which are displayed in a significant line of characters, at the left bottom of the abstract. (National Bureau of Standard, 1987)

\footnotetext{
"Fund item: This work was supported by Asia Research Center in Tsinghua University in 2018.

WANG Fengnian, Ph.D., Executive Editor, Editorial Department of Tsinghua University Journal, Tsinghua University.
} 
In 1999, editorial board issued Chinese Academic Journal (CD-ROM) Search and Evaluation Data Specification (CAJ-CDB/T1-1998). In 2000, the General Office of the Ministry of Education of People's Republic of China issued The Regulations for the Journal of Social Sciences in Colleges and Universities in China. In 2002, Academic Department of China Association for Science and Technology issued the Decision on Standardizing Keyword Selection in Academic Papers. All of these have made clear provisions on the indexing of keywords.

During the 1990s of 20th century, not only traditional information and informational science focused on the retrieval function of keywords, scholars of computer field also paid attention to the role of keywords in cyberspace. With the development of computer network and information retrieval system, the information retrieval function of keywords has entered the field of semi-automated modern computer science from the traditional library science direction.

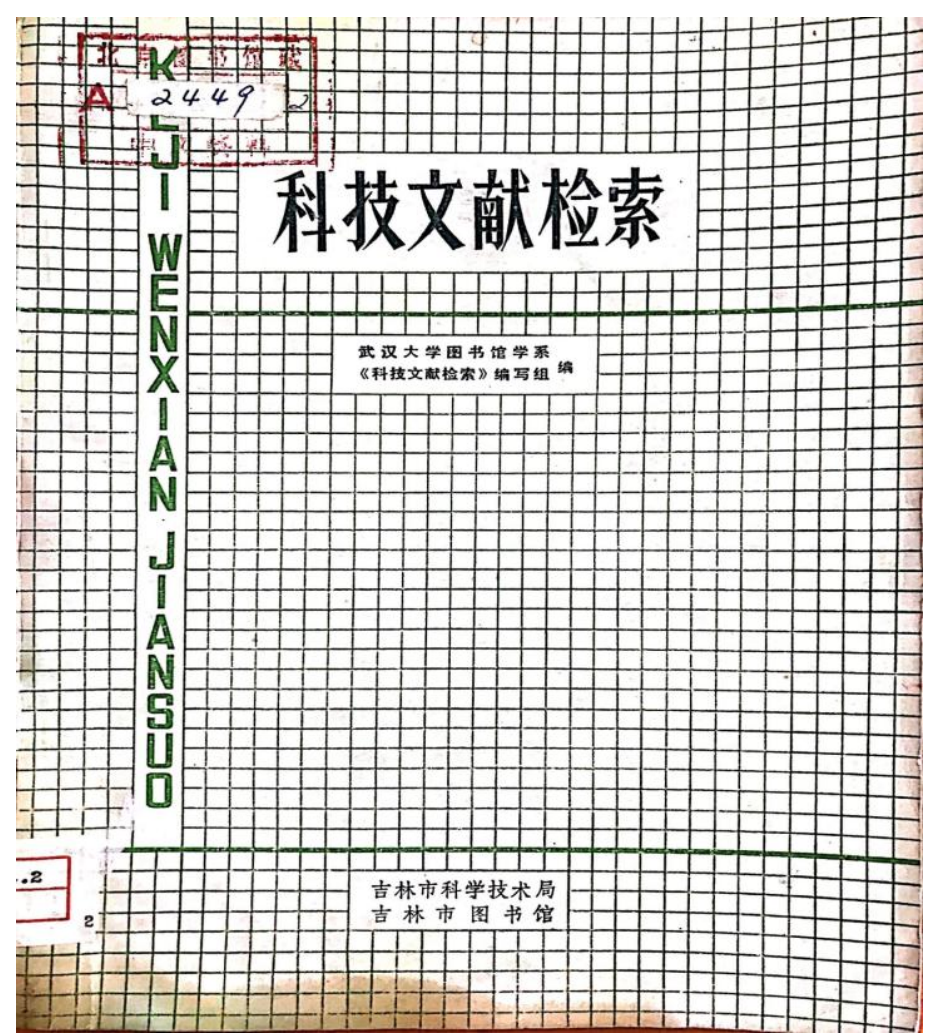

Figure 1. Bibliographic Search of Science and Technology.

\section{The Value of the Keywords in Academic Paper}

\section{Revealing the Gist of the Paper}

Keywords can express the theme of the paper clearly and intuitively. Readers can learn about the main idea of the paper at the first glance without reading the abstract or the text of the academic paper. For example, in the paper titled "Digital Journalism and Risk Communication Practice for Public Health Humanitarian Emergencies Response in Africa", the keywords include digital journalism; social media; resiliency humanitarian crisis; emergency; risk communication; mobile health; practice; Africa (Tambo, Kazienga, \& Fogang, 2017) which clearly indicated the main contents . 


\section{The Retrieval Value}

From the database perspective, journal papers are generally retrieved and indexed by the literature database, which is compiled according to the keywords provided by the academic papers. The keyword index of the literature database provides readers with a quick way to retrieve documents, so that they can find documents that meet their requirements quickly. Using keywords to search and query the latest published papers has become popular in the academic world.

In the Chinese literature search, keyword search is ranked after full-text search, topic search, title search, author search, and unit search, but in the actual search path, keyword search and topic search are used extensively. In the VIP Database (Chongqing VIP Database), the title/keyword is set as the default search format. For example, the keyword strategic industrialization is a combination of strategic and industrialization. When searching for strategic and industrialization separately, many materials closely related to the paper will be missed. Materials that are not related to strategic industrialization may be consulted; but conversely, if strategic industrialization was regarded as one of the keywords to search in the relevant literature, the useful material will be retrieved more accurately (Yan, 2015).

From the reader's point of view, with the increase of the types of academic journals and academic papers, the collection and reading of academic papers often appear dazzling, which makes many people in the reading of academic papers first look at the topic of the paper, and then browse the abstract and keywords, and make a search judgment. If the abstract and keywords of a paper are in line with readers' academic research needs, they will continue to read the whole paper.

\section{Enhancing the Influence of Academic Periodicals}

Because keywords are directly related to the probability of the retrieval of the paper and the utilization rate of the results of the paper, especially in the digital era, the influence of academic journals not only depends on the academic quality of the paper itself, but also relies on the CNKI (China National Knowledge Infrastructure), Wanfang Database, Longyuan Journal Net, CQVIP Company, and other digital platforms for promotion. For academic journals, increasing the probability of being retrieved from academic papers means increasing the probability that the academic paper will be cited, which means increasing the probability of enhancing the influence factors of academic journals. Therefore, scientific and reasonable keyword indexing can effectively enhance the influence factors of academic journals, so as to effectively enhance the ranking of academic journals in the evaluation system of academic journals and enhance their influence. Whether the keywords are well written or not directly affects the retrieval rate and citation rate, which can affect the academic status of academic journals and authors. The content of some academic papers has high academic values, but the improper setting of keywords leads to readers' misunderstanding and miscalculation of the academic impression of the paper, which lessens the reader's willingness to read, thus affecting the judgment of the academic value of the paper.

\section{Highlighting Specific Information}

Keywords will be highlighted in unique ways, such as changing the font, font size, bold and black, directly exclusive line, in a prominent position. Readers can quickly learn about the main contents of the paper, and make a decision to read it or not. 


\section{Generalizing Information}

In order to convey the important information of the paper in the fastest and most concise form, it is necessary to have a high degree of generalization and summary. For example, the paper, entitled "The Outline of Chinese Law - On the Knowledge, Axiology and Style Aesthetics of Chinese Civilized Legal Wisdom", covered 72 pages. However, from the five keywords: Chinese legal system; Chinese law; three dimensions and four principles; natural reason, humanity and national law; semantic system of legal meaning, readers can largely understand the context of Chinese jurisprudence (Xu, 2014).

\section{Problems Associated With Keywords in Academic Papers}

As an editor, I have not only found some keywords in unpublished papers to be problematic, but also, surprisingly, have come across improper keywords in published journals.

\section{Too Few Keywords}

Using too few keywords would not only cause inconvenience for readers who wish to know the main contents of a paper, but it might also be too general for readers who are searching for specific information.

In the paper titled Activities of Black Women's Clubs in America in Late 19th Century and Early 20th Century (Guo, 2008), there are only two keywords: black women's clubs; activities. From two keywords, readers are hardly able to search for the specific activities the Black Women's Clubs carried out. If the author added welfare; improving living conditions; sanitation; readers would be able to understand the main contents of the paper more clearly.

\section{Too Many Keywords}

Too many keywords, however, would undermine the process of guiding the general reader to relevant information. For example, there appear eight keywords in one paper: criminal law; criminal law theory; knowledge of illegality; intentional crime; negligent crime; formal illegality; essential illegality; social harmfulness (Ma \& $\mathrm{Hu}, 2008)$. If five of them, for example, criminal law; intentional crime; negligent crime; formal illegality; essential illegality, were deleted, and only three of them, for example, criminal law theory; knowledge of illegality; social harmfulness, would be easier for readers to search for the information they need without reading through unrelated content.

\section{Ambiguous Keywords}

Keywords should accurately reflect the full scope of the information in a paper. However, when words such as, research; studies; analysis; problems are used as keywords, the specific contents and purpose of the paper are not revealed. If such keywords are used, readers cannot know through a search the nature of the research that the paper contains.

For example, in one paper there are five keywords: Chuxiong City; Chenjiacaozi village; removal; strategy; research (Li, Luo, \& Wang, 2008). Two keywords of the five keywords, strategy; research, are too general: strategy, and research, without any specific relation to the content of the paper, can not lead readers to find what they are searching for in the paper. On the other hand, research; studies; analysis; problems are too general and should be deleted from the list of keywords in order not to leave readers in the dark concerning the exact content.

\section{Overlapping Keywords}

Some authors try to emphasize a certain issue by repeating certain words in their uses of keywords, which 
not only confuses the readers who wish to find specific information, but also hinders the reader's ability to comprehend the main content and purpose of the paper.

There are three keywords: reflective abilities; composition of reflective abilities; teacher culture in one paper (Yu, 2008), but two of them: reflective abilities; composition of reflective abilities with overlapping meanings.

\section{Keywords in a Colloquial Register}

Some authors use inappropriate non-academic register in their use of keywords. These expressions do not bear any significance to a reader who is searching for specific content. For example, as keywords, the expression Fast and Fine (Zhou, Liu, \& Wang, 2008) has no academic meaning, and is therefore not a suitable use of keywords for an academic paper.

\section{Lengthy Keywords}

Some authors use keywords in such a way as to express all the main ideas of a paper at great length. For example, one author chooses: set concept and non-concept, a juxtaposition of ideas in a single use of keywords (Shi \& Ruan, 2008). These two ideas should be separated into two sets of keywords, set concept and non-concept, instead of combining a single phrase.

\section{Reasons for Keyword Problems in Academic Papers}

\section{The Lack of Canonical Guidance}

At present, the main criteria or norms for the selection of keywords of academic papers, such as, Chinese Academic Journal (CD-ROM) Search and Evaluation Data Specification (CAJ-CDB/T1-1998), The Regulations for the Journal of Social Sciences in Colleges and Universities in China, Decision on Standardizing Keyword Selection in Academic Papers, etc., were mainly formulated in the early 21 th century. With the advent of the digital age and the prosperity of new disciplines and interdisciplinary disciplines, many of the above standards and norms were no longer applicable.

\section{Ignoring the Value of Keywords from Some Authors}

Some authors, when writing papers, tend to take more account of the academic views, academic quality, and other factors of the entire academic paper, while ignoring the value of keywords.

\section{Limitations of the Editorial Knowledge Structure}

Because some academic periodical editors are limited by objective factors such as subject background and subject knowledge, they often focus only on editing details such as typo, grammatical errors, and citation specifications, and some editors even lack the understanding of keyword indexing methods and do not make effective suggestions.

\section{Principles for Selecting Keywords in Academic Papers}

From the experience as an editor, I would suggest the following principles to reduce the problems listed above.

\section{Academic Principle}

Keywords should be academic rather than colloquial, in order to clarify the academic content of a paper.

\section{Accurate Principle}

Keywords should accurately reflect the main research areas and the purposes. It is essential to avoid using 
the keywords that may be misinterpreted.

\section{Logical Principle}

Keywords should be arranged in a logical way in relation to the content of the paper so as to correspond with the order in which the ideas are presented in the main body of the paper.

\section{Comprehensive Principle}

Keywords should express the full scope and purpose in order to be of practical use to the reader who is looking for specific content.

\section{Countermeasures of Selecting Keywords for Academic Papers}

\section{Timely Revising of the Keyword Selection Criteria}

Because the existing standards and norms for selecting keywords of academic papers cannot meet the needs of editing, therefore, it is necessary to revise and formulate reasonable keyword selection criteria and rules for academic papers. These standards and norms not only follow the norms of language and writing, but also take into account the specific requirements of related disciplines and editorial practice work, and truly make standards and norms actually refer to, which can be completed by the joint editorial researchers of academic journals, language and writing researchers, academic leaders of related disciplines.

\section{Improving the Author's and Editor's Awareness of Keywords}

Some editors just pay attention to the content, misprints, and punctuation of a paper whilst neglecting to check whether the keywords are appropriate. An author's primary concern is whether the paper will be published, and hence regards keywords as a minor detail. So from editors to authors, it is necessary for them to increase their awareness of the importance of keywords.

An effective way for readers to find specific information is to search for keywords. If authors and readers both would realize the importance of keywords for finding information, it would be an essential first step towards improving the quality of keywords.

\section{Improving the Professional Skills for Authors and Editors}

On June 1, 2008, The Regulations on the Administration of Professional Qualifications of Publishing Professional and Technical Personnel issued by the General Administration of Press and Publication of the Peoples' Republic of China, stipulated that "publishing professional and technical personnel should participate in continuing education for not less than 72 hours per year". Through the annual 72-hour training, editors should be familiar with the relevant standards and norms of editorial and publishing, and enhance the scientific selection ability including the keywords of academic papers. For example,

1. Selecting keywords from the headings at all levels. The headings at all levels illustrate what is described in the paper and contain many keywords. The subtitle of the paper is also to reflect the paper. The hierarchical titles of the topic contain useful keywords. For example, it is practical to extract three keywords, the commercial bibliographic databases; plight of the commercial bibliographic databases; strategies of the commercial bibliographic databases from the title of "The Predicament and Countermeasures of Commercial Bibliographic Databases" (Wang, 2017).

2. Selecting keywords from the abstract. The abstract contains the research methods, results, and conclusions of the paper, and contains many keywords. In general, the selection of keywords in the abstract can basically achieve the number of keywords necessary for the paper. 
3. Choosing keywords from introduction, material, method, and conclusion. In order to make up for some limitations of selecting keywords from the abstracts and titles, it is necessary to select keywords from the introduction, materials, methods and the conclusions of the paper.

\section{Using Peer-Review to Check the Keyword Quality}

In the peer-review process, the editorial department should not only evaluate the content of the manuscript, but also ask for feedback about keywords.

\section{Improving Communication Between Editors and Authors}

In many instances, authors were not aware that the keywords they used were problematic. Editors should report problems related to keywords to authors so that authors may revise their use of keywords accordingly.

\section{Putting the Requirements About Keywords in Journal Contributions}

Once a year, there are renewed journal contribution introductions for many journals. If the requirements about keywords were listed in journal contributions, authors would be clear how to choose keywords for academic papers.

\section{References}

Guo, G. L. (2008). Activities of Black Women's Clubs in America in late 19th century and early 20th century. Journal of China Women's University, 20(2), 79-81. [in Chinese]

Li, Z. R., Luo, W., \& Wang, Z. G. (2008). Analyses the city expanding stratagem of Chuxiong Prefecture based on displacement of Chen Jia Cao Zi Village. Journal of Chuxiong Normal University, 23, 96-102. [in Chinese]

Ma, R. C., \& Hu, X.-F. (2008). On knowledge of illegality in theory of criminal law. Journal of Henan University, 48(6), $20-23$. [in Chinese]

National Bureau of Standard. (1987). Format of scientific and technical reports, dissertations and academic papers. GBT 7713-1987. [in Chinese]

Shi, C. S., \& Ruan, L. P. (2008). The difference between set concept and non-set concept. Journal of Nanning Teacher College, 25(3), 78-79. [in Chinese]

Tambo, E., Kazienga, A., \& Fogang, M. (2017). Digital journalism and risk communication practice for public health humanitarian emergencies response in Africa. Journal of Mass Communication \& Journalism, 8(4), 383.

The Department of Science, Wuhan University Library. (1975). Bibliographic search of science and technology. Jilin: Jilin Science and Technology Bureau. [in Chinese]

Wang, F. N. (2017). The predicament and countermeasures of commercial bibliographic databases. Journalism and Mass Communication, 7(1), 17-24.

$\mathrm{Xu}, \mathrm{Z}$. Y. (2014). The outline of Chinese law_Knowledge, axiology and style aesthetics on the legal wisdom of Chinese civilization. Journal of Tsinghua University, 29(5), 5-72. [in Chinese]

Yan, P. (2015). Strategic industrialization: A new Lisztist industrialization theory. Journal of Tsinghua University, 30(5), $166-177$. [in Chinese]

Yu, Z. F. (2008). Considerations on teachers' reflective abilities. Journal of Yangzhou University, 12(6), 24-26. [in Chinese]

Zhou, J. B., Liu, H. B., \& Wang, G. L. (2008). Analysis on Ganzhou City's advantages of its fast and fine economic development. Journal of Shanxi Agricultural University, 7(2), 163-165. [in Chinese] 